\title{
PLANT VISUALIZATION USING IOT AND AR
}

\author{
Swapnil Likhitkar, Jatin Gereja, Nikhil Sonwane \\ School of Electrical Engineering, MIT Academy of Engineering, Pune, India \\ Savita Pawar \\ Assistant Professor, School of Electrical Engineering, \\ MIT Academy of Engineering, Pune, India
}

\begin{abstract}
The fourth industrial revolution has enabled entire manufacturing lines to operate with minimal human intervention. Machines have become autonomous and, for the efficient operation of systems, decision-makers require a clear picture of the plant's situation and overall performance. Sensors provide real-time machine data that can assist in understanding trends, enabling experts to analyze and optimize processes and also provides the ability to predict faults. Thus, providing access to plant data at the right level and time is critical for effective operations. Operators are required to quickly spot issues in real-time to take corrective measures which require effective delivery of information using specific Data Visualization techniques. This paper depicts a system developed by the integration of the latest technologies to virtually visualize the performance of an industrial plant for decision-guiding insights to reduce problems and improve production.
\end{abstract}

Keywords - Industry 4.0, Smart Factory, Internet of Things (IoT), Augmented Reality (AR), Predictive Maintenance.

\section{INTRODUCTION}

The terms "Industry 4.0" and "Smart Factory" are often associated with the concept of the Internet of Things (IoT), which refers to networked devices that can exchange data (De Pace et al, 2018). It is the way to collect, exchange, analyze and interpret data in real-time. This robust access to data enhances and improves operations. Another term Augmented Reality (AR) was officially coined by two scientists (Caudel and Mizell, 1992), employed at Boeing Corporation Tom Caudell and David Mizell, who developed an experimental AR system to simplify the manufacturing process of the air company in the twentieth century. Industrial augmented reality (IAR) is one of the key technology of the digitalization era, which connects workers with the physical world through overlaying digital information (Masood and Egger, 2019).

What if it was possible to combine the above two technologies? By overlaying machine data as digital content into a worker's physical world, they can become more effective at a variety of tasks, reducing unexpected equipment failure and improving manufacturing efficiency.

\section{LITERATURE SURVEY}

Early work in visualization techniques tended to take a sharply critical approach towards virtual customer experience, focusing on use cases predominantly found in Assembly, Manufacturing and Customized solutions. The research by (Jakl et al, 2018), highlights that the designed prototypes of Real-Time Machine Data Overlay were tested at a low level and required evaluation. Thus, they were considered experimental and were not used in real scenarios.

\section{Methodology}

The concept below is developed by integration of Augmented Reality and the Internet of Things to virtually visualize plant status using a scaled-down example. A general idea of the entire workflow is depicted in Fig. 1.

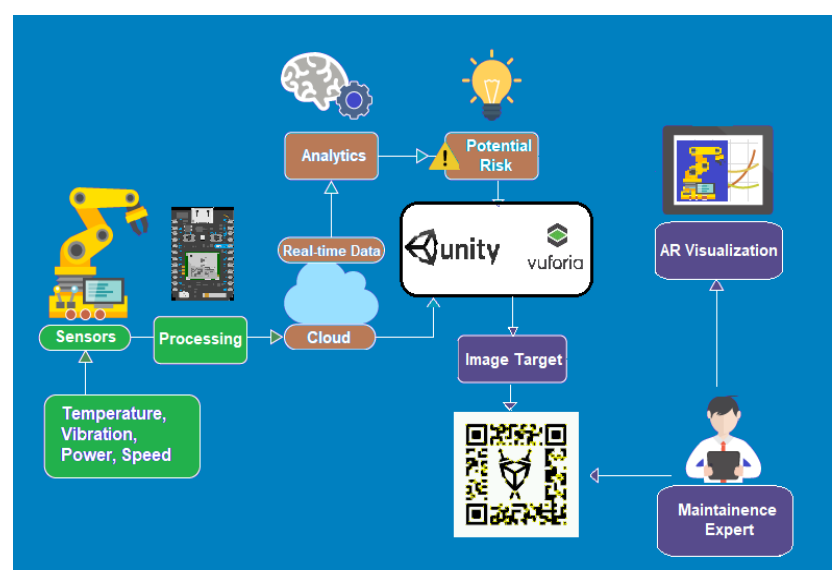

Fig. 1.System Workflow.

A conveyor is one of the primary systems found in any industrial plant. A conveyor system has a lot of parts but the most crucial part is the conveyor motor. Conveyor motors are under operation for long durations of time and may fail if not maintained properly. The maximum service life of any system depends on several critical parameters. Similarly, a motors life and performance depends on parameters like temperature, voltage, current, vibrations and, speed (Kamble and Patil, 2019). An RS775 PMDC motor represents the conveyor motor for the below designed system. The operating voltage of the 


\section{International Journal of Engineering Applied Sciences and Technology, 2020 \\ Vol. 4, Issue 12, ISSN No. 2455-2143, Pages 235-239 \\ Published Online April 2020 in IJEAST (http://www.ijeast.com)}

motor is $24 \mathrm{~V}$. The no-load current of the motor is $0.85 \mathrm{~A}$, and no-load speed is $12600 \mathrm{rpm}$. The weight of the motor is $0.3 \mathrm{Kg}$.

\section{A. Sensing}

The initial process of the system begins with the sensing unit. This stage highlights the significance of monitoring a machines critical parameters and the sensors required to record them with the example of RS775 motor.

1) Temperature of Motor Winding: Overloading of motor leads to increased motor current, resulting in a steady temperature rise. Exceeding the maximum temperature of magnets causes partial demagnetization and, permanently alters the performance of a motor (Montone, n.d). A TMP36 temperature sensor was used to monitor the temperature by positioning it close to the motor windings.

2) Voltage supply: Standard motor designs enable them to operate within $10 \%$ of rated voltage. Operation of the motor below this band decreases flux, badly affecting motor efficiency and torque. A 170640-B25 voltage sensor is used to monitor the voltage supplied to the motor. The sensor uses a voltage divider circuit for measuring the change in voltage.

3) Current drawn by the motor: Overcurrent occurs due to a low supply voltage or short-circuited conductors. As mentioned in (N, 2017) overcurrent condition leads to overheating of the motor and damages the insulation. An ACS712 current sensor was used to record the current drawn by the motor. As mentioned in the datasheet by (Allegro Microsystems, 2020), it is a fully integrated, Hall-effect based linear sensor with a $2.1 \mathrm{kV}$ RMS voltage isolation along with a low resistance current conductor.

4) Vibrations: (Bertolini and Fuchs, 2011) state that undesirable fluctuations like oscillating torques, superimposed on the desired motor torques result in oscillating rotational movements, leading to vibrations. Vibrations may create several mechanical issues inside a motor, affecting accuracy and efficiency. It can also accelerate wear and tear of moving parts. An SW-420 vibration sensor which has an LM393 comparator was used to record vibrations over set a threshold.

5) Motor speed: A rise in the motor speed beyond the maximum point increases the rate of change of voltage, resulting in severe sparking leading to commutator and brush wear and may also cause bearing failure. An LM393 speed sensor module was used to detect the speed. An encoder disc was mounted on the motor shaft and positioned between the groves of the sensor. The encoder disc has holes which obstruct the infrared light as per number rotations indicating speed.

\section{B. Processing}

The data recorded by sensors is raw and requires processing. Particle Photon controller is selected to carry out the processing task because of the features it offers. Particle photon, shown in Fig. 2, is an IoT hardware development board with $120 \mathrm{MHz}$ ARM Cortex M3 microcontroller named, STM32F205 (TechDesign, 2016) and a Broadcom Wi-Fi chip.

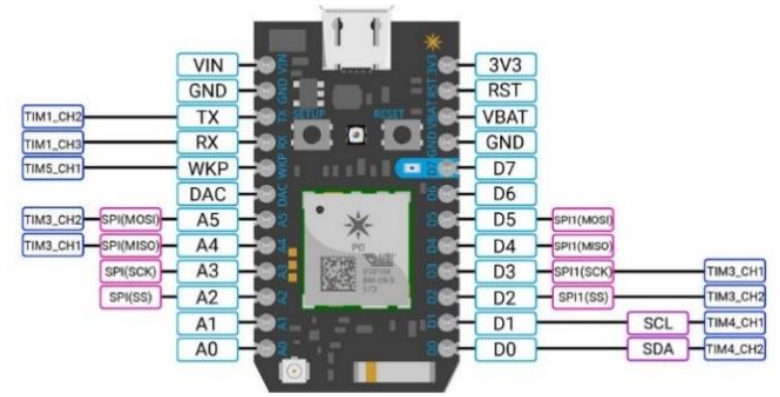

Fig. 2.Particle Photon Pin-Out.

Every message sent through Particle is encrypted and secure. Each device has a private key (API Key), to avoid unauthorized access to hardware (Particle, n.d). The device configuration closes all incoming ports to prevent active side attacks and port scanners. The radio connections are encrypted by industry-standard WPA2.

As shown in Fig. 3, a program is fed to the Particle Photon to carry out the processing task, enabling it to map sensor output voltage to a standard value. The sensor data is processed and uploaded to the designated section of the Particle cloud. The particle cloud provides a simple console and a dashboard option to view the data in multiple formats for statistical and analytical purposes.

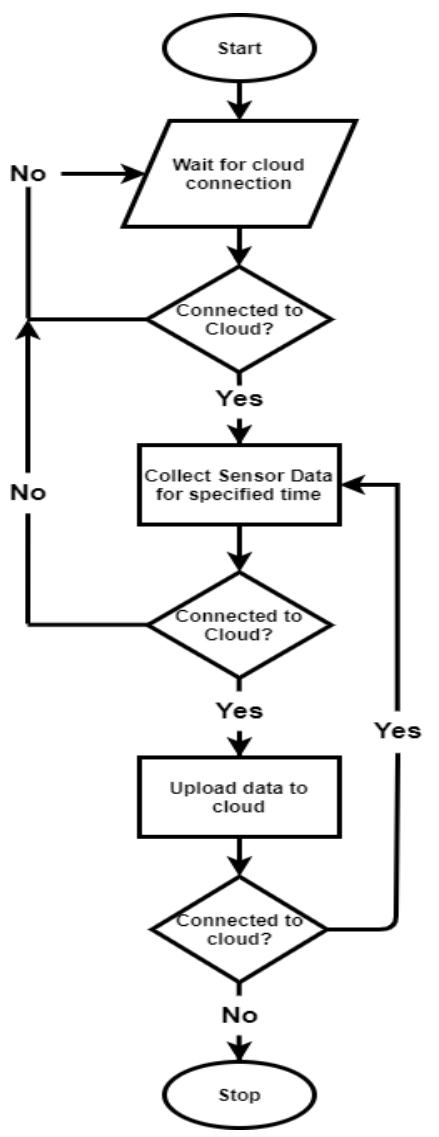

Fig. 3. Flowchart of Processing unit. 


\section{International Journal of Engineering Applied Sciences and Technology, 2020 \\ Vol. 4, Issue 12, ISSN No. 2455-2143, Pages 235-239 \\ Published Online April 2020 in IJEAST (http://www.ijeast.com)}

\section{Visualization}

The visualization unit is the final and the most crucial section of the entire process. The software used to achieve this task: Unity Engine and Vuforia engine.

The Unity engine is used to create virtual reality and augmented reality simulations. An Android/IOS compatible, Augmented Reality (AR) visualization application is designed and rendered in Unity. Fig. 4, shows the visualization design for the conveyor motor health.

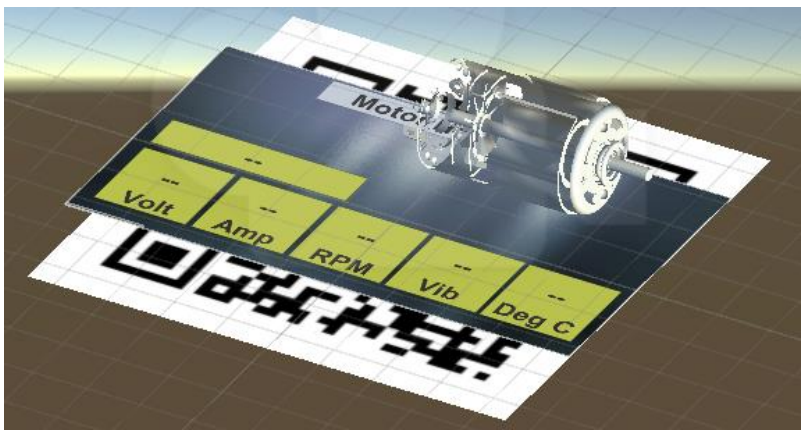

Fig. 4. Motor health card design developed in Unity Engine.

There can be several machines in a plant and, the AR visualization of sensor data will be different for all. The application must connect to a particular section of the cloud to see the parameters of a specific machine. Vuforia engine resolves this issue by use of "Markers" and "Image Targets." According to (Karthiga et al, 2018), Markers are the images that Vuforia uses as the points of reference to display our projections. They are unique and linked to only a single object. Image targets are the feature points extracted from the Markers. Here a QR code acts as the Marker and, is fed to Vuforia engines database. Vuforia first detects the image target, as shown in Fig. 5, and compares the "feature points" with the receiving frame of Marker through the device camera.

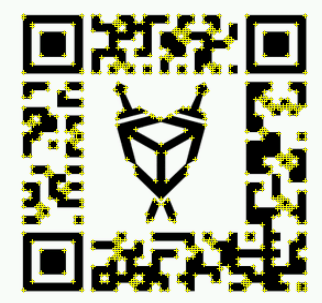

Fig. 5. Feature point detection using Vuforia Engine.

Upon a successful match, two backend programs fed in the AR application, fetch the data from the IoT device cloud by the use of an API Key. They set the bitrate of transmission, map the sensor data in the cloud to the tags provided in the AR application. The parameters are fetched as JSON files. These values are displayed in the user interface as augmented reality projections. The flowchart in Fig. 6, shows the software development process behind the visualization unit.

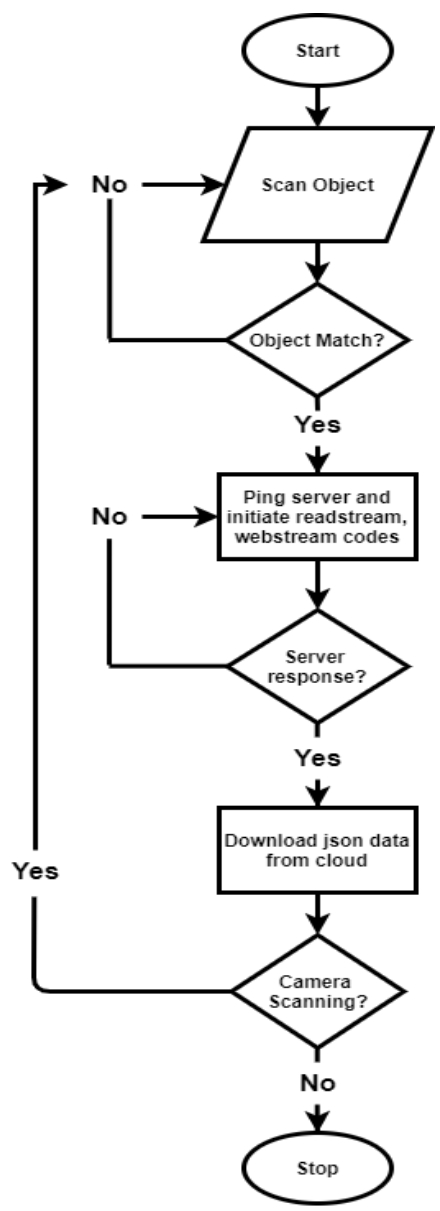

Fig. 6.Flowchart of Visualization unit

\section{Results And Discussions}

The final AR projection for conveyor motor health is depicted in Fig. 7. We programmed each parameter block to show a condition by the use of different colours. Green for stable operation, yellow depicts a dynamic value, blue for a low priority alert and, the orange colour signifies a high priority alert, meaning parameter beyond the safe limit.

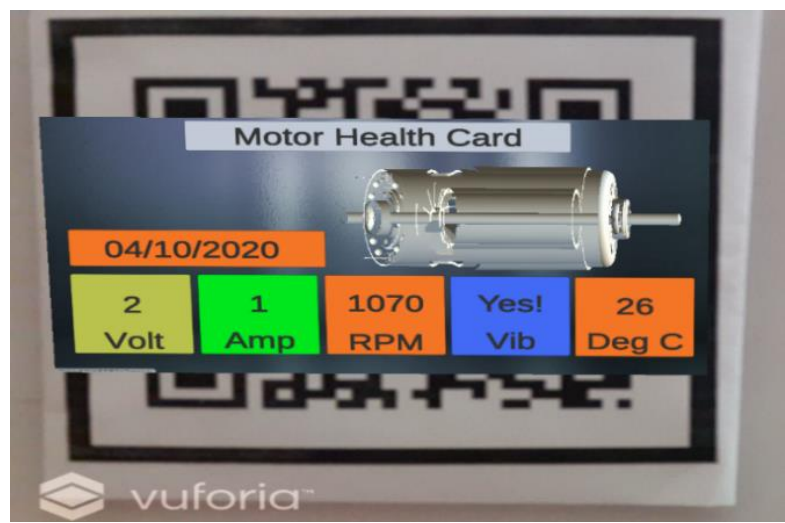

Fig. 7. Augmented Reality Projection of Motor health card. 


\section{International Journal of Engineering Applied Sciences and Technology, 2020 \\ Vol. 4, Issue 12, ISSN No. 2455-2143, Pages 235-239 \\ Published Online April 2020 in IJEAST (http://www.ijeast.com)}

This system can be used for visualizing the status of any electro-mechanical system in a factory. Fig. 8 highlights the system compatibility with different types of machinery.

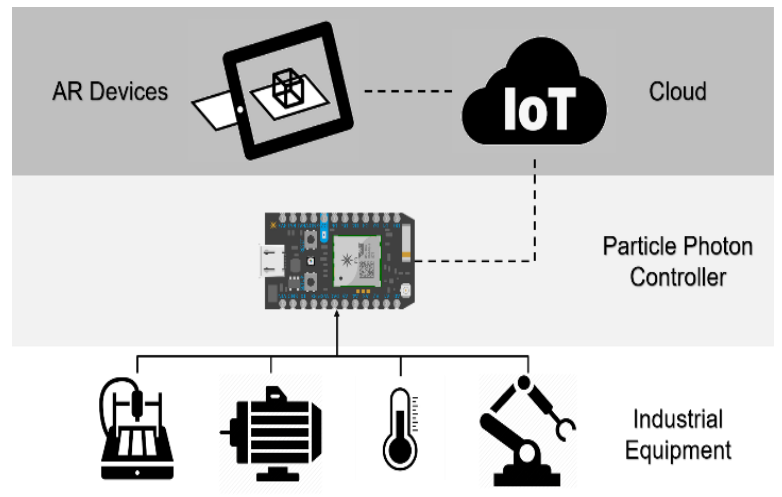

Fig. 8.Conceptual diagram of plant visualization.

It is easy to set up and requires only a few slight modifications. It can assist technicians to diagnose abnormal operation and assist them in finding out precise points of faults in machinery, improving the machine uptime and saving costs associated with repairs and production halt.

\section{CONCLUSION}

As stated by (Vaidya et al, 2018), Industry 4.0 defines a new level of organization and control over the entire value chain of the life cycle of products. As stated by (Siemens AG, n.d), Industry 4.0 is a trend towards digitalizing processes to improve manufacturing in terms of productivity, efficiency and flexibility. Millions of devices are bridging the real and the digital world and, transforming the way things were done.

The proposed system can provide a new level of control of an entire industrial plant by providing effective statistics and analytics on machines for active decision making. This system can be a remarkably productive method capable of bringing the control of an entire plant at those fingertips with a basic smartphone. It will be very beneficial, innovative and would incur much fewer expenses compared to other solutions.

\section{ACKNOWLEDGMENTS}

We would also like to thank Professor Mahesh Vibhute (MIT Academy of Engineering) and Professor Amit Nagarale (MIT Academy of Engineering) for their comments on an earlier version of the manuscript, although any errors are our own and should not tarnish the reputations of these esteemed persons.

We are immensely grateful to our Institute, MIT Academy of Engineering for providing us opportunities to gain industrial exposure without which we would not have got the chance to make this broad spectrum project.

\section{REFERENCES}

[1] De Pace, F., Manuri, F., and Sanna, A. (2018). Augmented Reality in Industry 4.0. American Journal of Computer Science and Information Technology. doi: 10.21767/2349-3917.100017.

[2] Caudell T., and Mizell D. (1992). Augmented reality: An application of heads-up display technology to manual manufacturing processes. Proceedings of the TwentyFifth Hawaii International Conference on System Sciences. doi: 10.1109/HICSS.1992.183317

[3] Masood, Tariq and Egger, Johannes. (2020). Adopting augmented reality in the age of industrial digitalisation. Computers in Industry. doi: 115. 103112. 10.1016/j.compind.2019.07.002.

[4] Jakl, A., Schöffer, L., Husinsky, M. and Wagner, M. (2018). Augmented Reality for Industry 4.0: Architecture and User Experience. 11th Forum Media Technology Conference. St. Pölten, Austria.

[5] Kamble, Indrani and Patil, Y. (2019). A Review of Parameters Monitoring and Controlling System for Industrial Motors using Wireless Communication. International Journal for Research in Applied Science and Engineering Technology. doi:10.22214/ijraset.2019.1010.

[6] Montone, Dan. (n.d). Temperature Effects on Motor Performance. Retrieved December 12, 2019 from https://www.haydonkerkpittman.com/-

/media/ametekhaydonkerk/downloads/whitepapers/temperature_effects_on_dc_motor_performance _1.pdf?la=en

[7] N, A. (2017). Causes of faults in electric motors and their effects. Electrical Equipment. Retrieved December 29, 2019 from http://engineering.electricalequipment.org/energy-efficiency-motors/causes-faultselectric-motors-effects.html

[8] Allegro Microsystems. (2020). ACS712. Retrieved $\begin{array}{llll}\text { February } & 9, & 2019 & \text { from }\end{array}$ https://www.allegromicro.com/en/Products/Sense/curre nt-sensor-ics/Zero-to-fifty-amp-integrated-conductorsensor-ICs/ACS712.aspx

[9] Bertolini, Thomas and Fuchs, Thomas. (2011). Causes of Vibrations and Noises. Vibrations and Noises in Small Electric Motors. Munich, Germany: Süddeutscher Verlag.

[10] TechDesign. (2016). Particle Photon's IoT Gene. Retrieved January 4, 2020 from https://blog.techdesign.com/particle-photons-iot-gene

[11] Particle. (n.d) Security Checklist for The Internet of Things: An essential guide to securing connected products. Retrieved January 4, 2020 from https://cdn2.hubspot.net/hubfs/2833873/White\%20Pape rs/security-3.pdf 
[12] Karthiga, V. G., Beniel, D., M. Aravind Kumar and S., Siva Shankar. (2018). Augmented Reality Game Development Using Unity and Vuforia. International Journal of Advance Engineering and Research. 5(3). 741-747.

[13] Siemens AG. (n.d). Industry 4.0. Retrieved February 26, 2020 https://www.plm.automation.siemens.com/global/en/ou r-story/glossary/industry-4-0/29278

[14] Vaidya, S., Ambad, P., and Bhosle, Santosh. (2018). Industry $\quad 4.0 \quad-\quad$ A Glimpse. doi: 10.1016/j.promfg.2018.02.034. 